\title{
MACHO RR LYRAE STARS IN THE GALACTIC BULGE: THE SPATIAL DISTRIBUTION
}

\author{
D. MINNITI, C. ALCOCK, D. ALVES, K. COOK, S. MARSHALL
} Lawrence Livermore National Laboratory

R. ALLSMAN, T. AXELROD, K. FREEMAN, B. PETERSON Mount Stromlo and siding Springs Observatory

A. RODGERS, K. GRIEST, M. LEHNER, T. VANDEHEI MSSSO and Univ. of California at San Diego
A. BECKER, M. PRATT, C. STUBBS, A. TOMANEY
University of Washington
AND
P. QUINN, D. BENNETT, W. SUTHERLAND, D. WELCH ESO, Notre Dame, Oxford, and McMaster

We have analyzed a sample of 1150 type ab, and 550 type c RR Lyrae stars found in 24 of 94 bulge fields of the MACHO database. These fields cover a range in Galactocentric distances from 0.3 to $1.6 \mathrm{kpc}$. In combination with the data on the outer bulge fields of Alard (1997) and Wesselink (1987), here we present the surface density distribution of bulge RR Lyrae between 0.3 and $3 \mathrm{kpc}$.

The distribution along the line of sight of the bulge RR Lyrae population was examined on the basis of the mean magnitudes, and it was shown that the bulk of the RR Lyrae population is not barred (Alcock et al. 1998). There is a hint of a barred distribution only in the RR Lyrae of the inner fields closer to the Galactic center $\left(l<4^{\circ}, b>-4^{\circ}\right)$. The red giant clump stars in the MACHO fields, however, clearly show a barred distribution, confirming the results of previous studies (e.g. Dwek et al. 1995, Stanek et al. 1996).

In the MACHO fields studied there are about 550 clump giants per RR Lyrae star. The RR Lyrae trace metal-poor stars, which are a minor component of the bulge population. The clump giants, however, should 
trace the bulk of the metal-rich population, following underlying mass of the bulge more closely

Given the different spatial distribution, we concluded that the RR Lyrae and the clump giants trace two different populations (Alcock et al. 1998). The RR Lyrae would represent the inner extension of the Galactic halo in these fields (Minniti 1996).

The observed surface distribution of RR Lyrae in the bulge fields was computed after discarding background RR Lyrae that belong to the Sgr dwarf galaxy (Alard 1996, Alcock et al. 1997). This distribution, shown in Fig. 1, yields a power law density distribution of the form $\rho=\rho_{0} r^{-3.0}$. There is no turnover or flattening of this distribution even in the innermost fields, indicating that the RR Lyrae population is very concentrated, with core radius $R_{c}<0.5 \mathrm{kpc}$. We also determine that the $\mathrm{RR}$ Lyrae surface distribution in the bulge fields is flattened, with $b / a=0.7$.

(Work at LLNL supported by the US DOE under contract W-7405-Eng-48.)

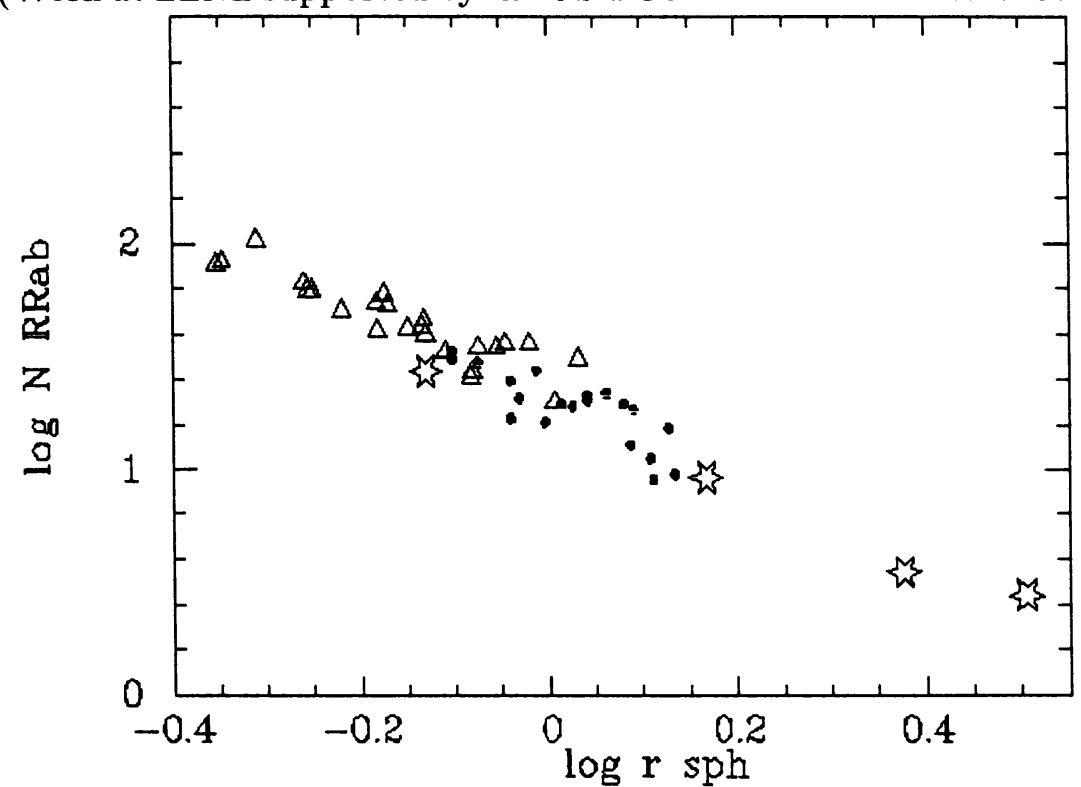

Fig. 1 - Surface density distribution of RR Lyrae type ab in the bulge from Alard (1997) -full circles-, from MACHO -open triangles-, and from Wesselink (1987) -stars-. The RR Lyrae of the Sgr dwarf galaxy are not included in these counts.

\section{References}

Alard, C. 1996, ApJ, 458, L17

Alcock, C., et al. (The MACHO Collaboration) 1997, ApJ, 474, 217

Alcock, C., et al. (The MACHO Collaboration) 1998, ApJ, Jan 1 issue

Dwek, E., et al. 1995, ApJ, 445, 716

Minniti, D. 1996, ApJ, 459, 175

Stanek, K. Z. 1996, ApJ, 460, L37

Wesselink, Th. J. H. 1987, Ph.D. Thesis, Katholieke Universiteit te Nijmegen 